\title{
Erratum to: Confinement versus interface bound states in spin-orbit coupled nanowires
}

\section{Lorenzo Rossi, Fabrizio Dolcini ${ }^{\mathrm{a}}$ (D), Fausto Rossi}

Dipartimento di scienza Applicata e Tecnologia, Politecnico di Torino, Torino, Italy

Published online: 14 October 2020

(C) Società Italiana di Fisica and Springer-Verlag GmbH Germany, part of Springer Nature 2020

Publisher's Erratum to: Eur. Phys. J. Plus (2020) 135:597 https://doi.org/10.1140/epjp/s13360-020-00614-2

During production, mistakes have been introduced.

1) On page 3, section 2.1, line 10, the expression "Zeeman-dominated" is correct, instead of "Rashba-dominated".

2) On page 3, section 2.1 , lines 11 and 12 , in the equation the less-than resp. greater-than signs are corrected.

3) On page 3, section 2.1, line 11, the expression 'Rashba-dominated" is correct, instead of "Zeeman-dominated".

4) On page 7,12th line after equation (12), in both terms the $Z$ as subscript is correct. The original article has been corrected.

The original article can be found online at https://oi.org/10.1140/epjp/s13360-020-00614-2.

\footnotetext{
a e-mail: fabrizio.dolcini@polito.it (corresponding author)
} 